OPEN ACCESS

Edited by:

Yi Wang,

Zhejiang University, China

Reviewed by:

Simone Carradori,

University "G. d'Annunzio" of Chieti-

Pescara, Italy

Yuelin Song,

Beijing University of Chinese Medicine,

Chu Chu,

Zhejiang University of Technology,

China

*Correspondence:

$\mathrm{Jin} \mathrm{Li}$

Lijin@tjutcm.edu.cn

Yan-xu Chang

tcmcyx@tjutcm.edu.cn

tThese authors have contributed equally to this work

Specialty section: This article was submitted to Ethnopharmacology,

a section of the journal

Frontiers in Pharmacology

Received: 02 August 2020 Accepted: 08 October 2020 Published: 30 October 2020

Citation:

Yang $X$, Sun A, Boadi EO, Li J, He J, Gao X and Chang Y (2020) A Rapid High Throughput Vibration and VortexAssisted Matrix Solid Phase Dispersion for Simultaneous Extraction of Four Isoflavones for Quality Evaluation of Semen Sojae Praeparatum. Front. Pharmacol. 11:590587. doi: 10.3389/fphar.2020.590587

\section{A Rapid High Throughput Vibration and Vortex-Assisted Matrix Solid Phase Dispersion for Simultaneous Extraction of Four Isoflavones for Quality Evaluation of Semen Sojae Praeparatum}

\author{
Xuejing Yang ${ }^{1,2+}$, Ali Sun ${ }^{1,3+}$, Evans Owusu Boadi ${ }^{1,3}$, Jin $\mathrm{Li}^{1 *}$, Jun $\mathrm{He}^{1,3}$, Xiu-mei Gao ${ }^{1}$ and \\ Yan-xu Chang ${ }^{1,3 *}$ \\ ${ }^{1}$ State Key Laboratory of Component-based Chinese Medicine, Tianjin University of Traditional Chinese Medicine, \\ Tianjin, China, ${ }^{2}$ School of Pharmacy, Harbin University of Commerce, Harbin, China, ${ }^{3}$ Tianjin Key Laboratory of \\ Phytochemistry and Pharmaceutical Analysis, Tianjin University of Traditional Chinese Medicine, \\ Tianjin, China
}

Isoflavones (daidzein, daidzin, genistein and genistin) were main chemical components and usually selected as markers for quality control of Traditional Chinese Medicine semen sojae praeparatum (SSP). High throughput vibration and vortex-assisted matrix solid phase dispersion and high performance liquid chromatography with diode array detection were developed to simultaneously extract and quantify four isoflavones in SSP. Some parameters influencing extraction efficiency of isoflavones by vortex-assisted matrix solid phase dispersion such as sorbent type, ratio of sample to sorbent, crushing time, vibration frequency, methanol concentration, eluting solvent volume and vortex time were optimized. It was found that the best extraction yields of four isoflavones were obtained when the sample $(20 \mathrm{mg})$ and SBA-3 $(40 \mathrm{mg})$ was crushed by ball mill machine for 2 min at vibration frequency of 800 times per minute. Methanol/water $(1.5 \mathrm{ml}, 8: 2, \mathrm{v} / \mathrm{v})$ solution was dropped into the treated sample and vortexed for $3 \mathrm{~min}$. The recoveries of the four isoflavones ranged from 86.1 to $94.8 \%$ and all relative standard deviations were less than $5 \%$. A good linearity $(r>0.9994)$ was achieved within the range $0.5-125 \mu \mathrm{g} / \mathrm{ml}$. It was concluded that the high throughput vibration and vortex-assisted matrix solid-phase dispersion coupled with high performance liquid chromatography was user-friendly extraction and quantification method of multiple isoflavones for quality evaluation of SSP.

Keywords: isoflavones, high throughput ball mill, vibration and vortex-assisted MSPD, semen sojae praeparatum, high performance liquid chromatography 


\section{INTRODUCTION}

Traditional Chinese medicines (TCMs) have been used to treat the diseases for thousands of years. Biological activities of TCMs with therapeutic or toxic effect largely attributed to multiple effects of numerous components in TCMs (Yang et al., 2011). From this perspective, the great attention should be paid to simultaneous extraction and purification of target components from TCM samples for purposes of guaranteeing their efficacy and safety. Furthermore, the procedure for sample preparation is a high-priority step in analytical methods owing to the complexity of TCMs matrices and relatively low active components in TCMs. Conventional sample pretreatment techniques for extracting components in TCM include ultrasonic extraction, heat reflux extraction, soxhlet extraction, solid phase extraction, liquid-liquid extraction and microwaveassisted extraction (Yang et al., 2011; Xiong et al., 2009; Wu et al., 2012; Xiong et al., 2012; Yue et al., 2018; Akbari et al., 2019). However, these procedures are usually time consuming, inefficient, costly and require large volumes of organic solvents. In view of these shortcomings, it is invaluable to develop a simple, green and efficient pre-concentration method for components analysis of TCMs.

Semen sojae praeparatum (SSP) is a famous TCM for treating febrile fever, cold fever, headache, irritability and chest tightness in the Chinese pharmacopoeia (Chinese Pharmacopoeia Commission, 2020). It is made from fermented matured seed of soybean (Glycine max (L.) Merr). It was also regarded as an important food material of the traditional Chinese diet by Chinese communities around the world (Guo et al., 2018). According to the Classic books of TCM "Jin-Kui-Yao-Lue" and "ShangHanLun," SSP was the main component of classical formula "Zhi-Zi-Da-Huang Tang," "Zhi-Shi-Zhi-Zi-Chi-Tang," and "Zhi Zi Chi Tang," which have been clinically utilized for the treatment of alcoholic hepatitis, abdominal distension and depression for more than thousand years (Qu et al., 2014; Chen et al., 2015; Wang et al., 2016; Wang et al., 2019). It was reported that SSP could reduce oxygen consumption in the heart muscle, improve microcirculation, and treat tumors and osteoporosis (Qu et al., 2007; Chai et al., 2019). Genistein, genistin, daidzin, and daidzein were the main isoflavones of SSP due to its extensive biological activities. Isoflavones were known as natural phytoestrogens because their structure is similar to the structure of estradiol. They could simulate the bidirectional regulation of endocrine levels (Chai et al., 2017; Yao et al., 2018). Phytoestrogens, especially distributed in legumes, could replace the role of estrogen and prevent the occurrence of adverse reactions to estrogen. Experience in TCM shows that the herbal medicine containing isoflavones were often used to treat the depressurization, hypoglycemic, lipid-lowering, prevent disease of heart head blood-vessel and atherosclerosis, etc. Phytoestrogens in prevention and treatment of menopausal syndrome, osteoporosis after menopause, etc., are similar to the positive role of estrogen replacement therapy (Vitale et al., 2013; Cederroth and Nef, 2009). Therefore, it was meaningful to develop the efficient extraction and quantitative determination method of these bioactive isoflavones for improvement of quality evaluation of SSP (Mocan et al., 2018).
Matrix solid-phase dispersion (MSPD) was proposed and pioneered by Barker (1989), which was a robust technology for simultaneous extraction and clean-up of target components from solid or semi-solid matrices in a single step (Barker et al., 1989). It has been widely applied to detection of various target components in plant matrices, animal tissues, food stuff, sludge, aquatic biota, soil and indoor dust samples (Dorota and Marta, 2019). MSPD is a modified solid-phase extraction (SPE) method that requires manual blending of sample with solid abrasive material before elution of target components with fitting solvent. This technique is too cumbersome to enhance efficiency as well as simplify the analytical procedures. Vortexassisted MSPD (VA-MSPD), which is based on substitution of the SPE step for a vortex agitation procedure, has demonstrated satisfactory results (Caldas et al., 2013; Escarrone et al., 2014; Hertzog et al., 2015; Du et al., 2018). Subsequently, a vortexhomogenized MSPD (VH-MSPD) has been established to determine halogenated phenolic compounds in seafood (Chen et al., 2016a) and short chain chlorinated paraffin from indoor dust samples (Chen et al., 2016b). This modified MSPD used vortex agitation procedure as mixing and blending steps instead of mortar and pestle for blending and homogenization. Recently, a combination of these two simplified techniques termed as dualvortex-assisted MSPD, has been used to determine nine parabens in door dust (Chung et al., 2019). In spite of these improvements which made operation more convenient, the aforementioned techniques require transfer procedure between extraction (blending) and clean-up (the SPE) steps, which may result in quantitative loss of analytes. Subsequently, new procedure, ballsin-tube MSPD was proposed and used to determine 133 pesticide residues in apple, peach pear and plum. In this method, all sample preparations were done directly in closed extraction tube with the assistance of steel balls (Kemmerich et al., 2019). Preprocessing process of balls-in-tube MSPD required manual operation which is less efficient and time consuming especially when large batch of samples need be processed. Accordingly, the more robust, high-efficiency and controllable blend method is in great request.

Vibrating ball mill also known as tissuelyser or mixer mill is an equipment with two high-speed large-amplitude arms which grinds, mixes and breaks cells in seconds or minutes through grinding balls impacting and friction within the bowl. It is suitable for milling and homogenizing soft, fibrous, hard and brittle materials in the wet and dry state. Various kinds of samples ranging from plants, food, tissue of human and animals, hair have been crushed using vibrating ball mill (Sun et al., 2018). In other literatures, sea foods and human hair were crushed with ball mill but grinding was manually done with mortar and pestle (MoredaPineiro et al., 2012; Miguez-Framil et al., 2013). The extraction (blending) step was accomplished by substituting manual grinding with ball milling.

In present study, it aimed at optimizing a rapid, high throughput MSPD technique for detection of genistein, genistin, daidzein and daidzin in SSP. A modified MSPD method named as high throughput vibration and vortexassisted matrix solid phase dispersion (VVA-MSPD) was proposed and established to extract and determine four 
isoflavones in SSP. All operations were performed in the same extraction tube in high throughput with ball mill machine vibration process. The vortex agitation was used to replace mixing, blending and SPE step, respectively. Besides minimization of manual grinding, the loss of analytes arising from transfer of mixed powder to SPE column or tube was reduced. Furthermore, ball mill has ability to handle dozens of simultaneous samples within seconds or a few minutes in which it might take hours by manual operation with great care and consistency. Additionally, main parameters affecting VVAMSPD were systematically investigated to optimize this new method. In a nutshell, the newly established VVA-MSPD coupled with high performance liquid chromatography (HPLC) was successfully used to extract and detect the isoflavones for quality evaluation of SSP (Figure 1).

\section{MATERIALS AND METHODS}

\section{Chemicals and Reagents}

HPLC grade methanol and formic acid were purchased from Fisher (Leicestershire, United Kingdom). Genistein, genistin, daidzein and daidzin (purity on HPLC > 98\%) were supplied by Chengdu Desite Bio-Technology (Chengdu, China). C18 and Silica were provided by Welch Materials (Chatham Road Ellocott City, United States). SBA-3, TS-1 and MCM-41 were obtained from Nanjing JCNANO Technology (Nanjing, China). Ultrapure water was purified by a Grindi-Q Academic ultra-pure water system (Grindipore, Milford, MA, United States). All other reagents were of analytical grade.

\section{Herbal Plant}

Five batches of SSP samples were obtained from the local pharmacy shops. All samples were identified as fermented processed product of matured seed of soybean [Glycine max (L.) Merr] by Prof. Yanxu Chang (Tianjin University Traditional Chinese Medicine). The material was ground through 65 mesh sieve, dried at $40^{\circ} \mathrm{C}$ and stored in a dryer.

\section{Apparatus and HPLC Analysis}

Blending was done with a ball mill machine (TJG-25, Techin, China) with an adapter of $2 \times 24$ well plate that was fixed by fastening device, ceramic beads of $5 \mathrm{~mm}$ diameter and $2.0 \mathrm{ml}$ micro-centrifuge tube (Beijing Labgic Technology Co., Ltd., Beijing, China).

Analysis was carried out on Agilent 1260 system (Agilent, Santa Clara, CA, USA) coupled with photodiode array detector (Scanning range $210-400 \mathrm{~nm}$ ). Separation was performed on an Agilent Eclipse Plus C18 column $(4.6 \mathrm{~mm} \times 100 \mathrm{~mm}, 1.8 \mu \mathrm{m})$ connected with a LC-18 guard column $(4.6 \mathrm{~mm} \times 12.5 \mathrm{~mm}, 5 \mu \mathrm{m})$ at $30^{\circ} \mathrm{C}$. Linear gradient elution system with acetonitrile (A) and ultrapure water (B) was as follows: $5-20 \%$ A at $0-10 \mathrm{~min}, 20-25 \%$ $\mathrm{A}$ at $10-25 \mathrm{~min}, 25-45 \% \mathrm{~A}$ at $25-32 \mathrm{~min}, 45-62 \% \mathrm{~A}$ at $32-37 \mathrm{~min}, 62-95 \% \mathrm{~A}$ at $40-45 \mathrm{~min}$. Flow rate was set at $0.3 \mathrm{ml} / \mathrm{min}$, injection volume of $2 \mu \mathrm{L}$ for each run and detection wavelength of $258 \mathrm{~nm}$.

\section{Preparation of Standard Solutions}

Concentration of $1 \mathrm{mg} / \mathrm{ml}$ of each isoflavone (genistein, genistin, daidzein, and daidzin) in methanol solution $(60 \%, \mathrm{v} / \mathrm{v})$ was prepared. In addition, a mixed standard solution containing $0.25 \mathrm{mg} / \mathrm{ml}$ of each isoflavone was prepared and further diluted into range of concentrations for calibration curves.

\section{Vibration and Vortex-Assisted MSPD Procedure}

Twenty milligram pulverized SSP and $40 \mathrm{mg}$ sorbent (C18, silica, MCM-41, SBA-3 and TS-1) were mixed in a $2 \mathrm{ml}$ round bottom centrifuge tube. A small ceramic ball was added and the lid covered tightly. The centrifuge tube was put into the adapter and installed on the ball mill machine to homogenize the mixture. Vibration frequency and crushing time were set at 800 times per min and 2 min, respectively. Ceramic ball was removed from the tube after the power settled.

One and an half milliliter methanol/water $(8: 2, \mathrm{v} / \mathrm{v})$ was gently dropped into the tube and vortexed for $3 \mathrm{~min}$. Thereafter, the solution was centrifuged at $550 \mathrm{~g}$ for $10 \mathrm{~min}$. The eluted solvent was then filtered with $0.22 \mu \mathrm{m}$ nylon membrane before HPLC analysis.

\section{Vibration-Forced MSPD Procedure}

Initial procedures were same as outlined in VVA-MSPD method ("Vibration and Vortex-Assisted MSPD Procedure" section). After removal of ceramic ball, the mixture was transferred into an SPE column with a sieve plate at the bottom. Another sieve plate was gently blocked on the top with a glass rod. Methanol/water (8:2, $\mathrm{v} / \mathrm{v}$ ) was dropped into the tube to elute the volume to $1.5 \mathrm{ml}$ after connection with a vacuum pump. The elute solvent was centrifuged at $550 \mathrm{~g}$ for $10 \mathrm{~min}$ and filtered with $0.22 \mu \mathrm{m}$ nylon membrane before HPLC analysis.

\section{Normal Vortex-Assisted MSPD Procedure}

Instead of ball milling procedure, $20 \mathrm{mg}$ pulverized SSP and $40 \mathrm{mg}$ sorbent (SBA-3) were mixed in an agate mortar, ground for 2 min to attain a homogenous mixture and then transferred into a centrifuge tube. Subsequent procedures were same as outlined in VVA-MSPD method.

\section{Ultrasonic Extraction}

According to a previous study (Niu et al., 2010), $0.500 \mathrm{~g} \mathrm{SSP}$ was ultrasonically extracted ( $40 \mathrm{kHz}, 96 \%$ power) with $20 \mathrm{ml} 50 \%(\mathrm{v} / \mathrm{v})$ aqueous ethyl alcohol for $50 \mathrm{~min}$. Thereafter, the lost weight was replenished with aqueous ethyl alcohol $(50 \%$, v/v) until reaction cooled down to room temperature. The solution was then filtered with $0.22 \mu \mathrm{m}$ pore membrane before HPLC analysis.

\section{Heating Reflux Extraction}

Heating reflux extraction was also performed in accordance with a previous study (Chai et al., 2014). Briefly, $1.000 \mathrm{~g} \mathrm{SSP}$ was mixed with $25 \mathrm{ml} 75 \%(\mathrm{v} / \mathrm{v})$ aqueous methanol solution in a conical flask. The mixture was weighed, heat refluxed for $30 \mathrm{~min}$, cooled down to room temperature and reweighed. Lost weight was made with $75 \%$ methanol $(\mathrm{v} / \mathrm{v})$. The solution was then shaken and 
transferred to a $25 \mathrm{ml}$ volumetric flask and then brought to volume with $75 \%(\mathrm{v} / \mathrm{v})$ aqueous methanol. The solution was filtered through a $0.22 \mu \mathrm{m}$ pore membrane prior to chromatographic analysis.

\section{Optimization of VA-MSPD Parameters}

In order to obtain higher precision, sensitivity and efficiency of extracting isoflavones from SSP, seven parameters which can influence VVA-MSPD procedure were optimized. Specifically, type of sorbent (C18, silica, MCM-41, SBA-3 and TS-1); ratio of sample to sorbent $(1: 0,2: 1,1: 1,1: 2$, and 1:3); crushing time of ball mill (1, 2, 3, and $4 \mathrm{~min})$; vibration frequency of ball mill $(500,800$, and 1,000 times per min); concentration of methanol $(50,60,70$, $80,90$, and $100 \% \mathrm{v} / \mathrm{v})$; volume of eluting solvent $(0.5,0.75,1,1.25$, 1.5 , and $1.75 \mathrm{ml})$; and vortex time $(1,2,3$, and $4 \mathrm{~min})$ were investigated. Each optimized parameter was further analyzed. All analytical samples were also analyzed in triplicate.

\section{RESULTS AND DISCUSSION}

\section{Effect of Related Factors in MSPD Types of Sorbent}

Sorbent type is usually the main factor influencing sensitivity and selectivity of extraction the pre-analytical stage. Normal-phase material (silica), reversed-phase material (C18) and three molecular sieves (MCM-41, SBA-3 and TS-1) were selected to optimize the preferred sorbent. As shown in Figure 2A, the efficient of $\mathrm{C} 18$ was the least when the same ratios of liquid to solid remained constant. Comparatively, efficiencies of MCM-41, TS- 1 and silica were similar whereas the efficient SBA- 3 was the most in extracting isoflavones. SBA-3 was the preferred sorbent for further MSPD extraction procedure. SBA-3 has been applied in separation and preconcentration of crystal violet in water sample by a solid phase preconcentration procedure (Azarkohan et al., 2013). However, it has not been used in plants samples and MSPD process. This study indicated that SBA-3 was a suitable molecular sieve sorbent for MSPD procedure. Molecular sieves are crystalline metal aluminosilicates comprising of three-dimensional interconnecting networks of tetrahedral oxides.

\section{Mass Ratio of Sample to Sorbent}

Mass ratio is related to both interface area of sample matrix and dispersant as well as elution process. Results shown in Figure 2B indicated that peak areas of each target isoflavone increased gradually as mass of SBA-3 increased from 0 to $40 \mathrm{mg}$ (1:0-1: $2)$. With increased amount of SBA-3, more surface area and crystal cavities are available to target isoflavone. Interaction between sample matrix and sorbent gets higher, however, extraction yields of target isoflavone were slightly lower at ratio of sample to sorbent (1:3). Excess sorbent may lead to incomplete elution for the four isoflavones. In this regard, optimal sample to sorbent ratio of 1:2 was selected for further tests.

\section{Crushing Time of Ball Mill}

Crushing procedure supplied external forces to pulverize samples and facilitated interactions between samples and sorbents. Results presented in Figure 2C showed that the highest extraction efficiency was achieved for 2 min crushing time whereas prolonged crushing (from 3 to $4 \mathrm{~min}$ ) gradually decreased yields of all isoflavones. Increment of crushing time may cause stronger interaction force between the target isoflavones and the dispersing sorbents, which make elution more difficult. Crushing time of $2 \mathrm{~min}$ was adapted for subsequent analysis.

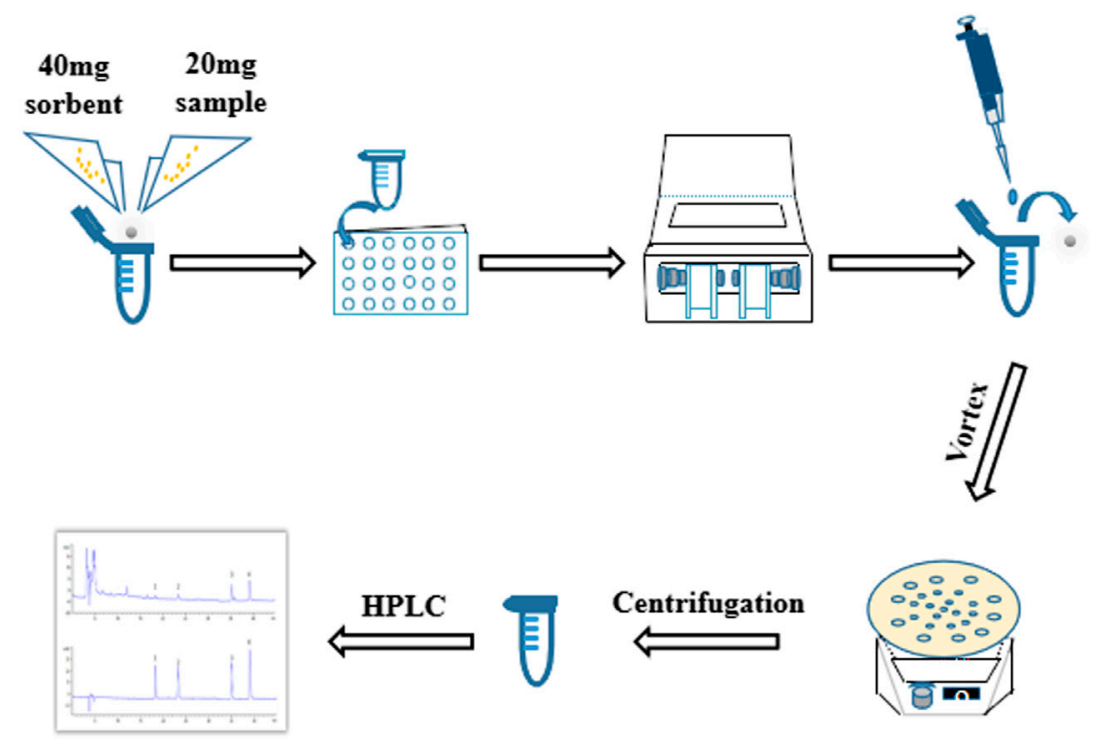

FIGURE 1 | Schematic diagram of vibration and vortex-assisted MSPD method. 

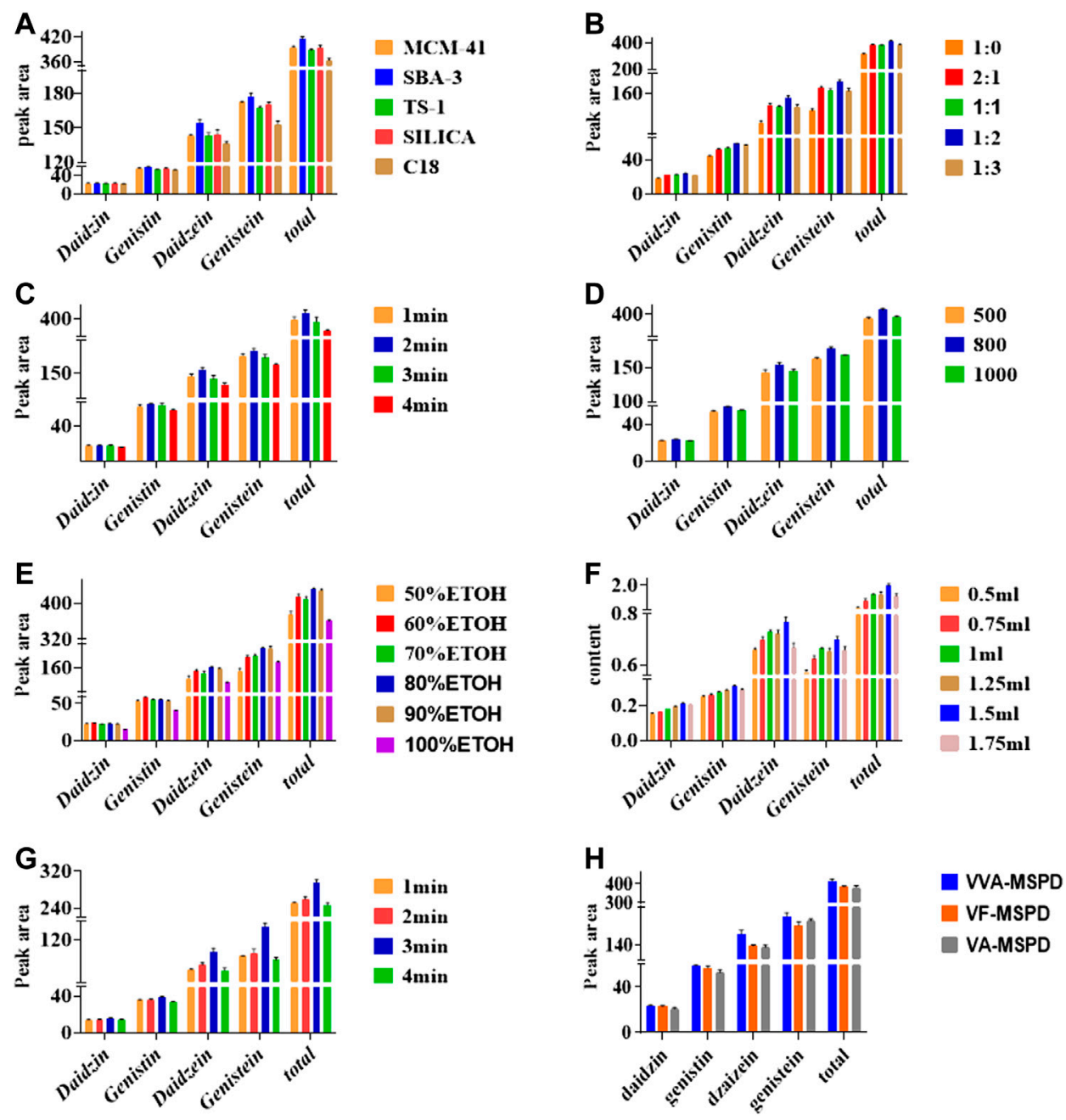

FIGURE 2 | Effects of parameters on extraction of four compounds by single factor using WA-MSPD technique: (A) type of sorbent, (B) mass ratio of sample to sorbent, (C) crushing time of ball mill, (D) vibration frequency of ball mill, (E) Selection of Methanol with different concentration, (F) volume of eluting solvent, (G) vortex time, $\mathbf{( H )}$ comparison of WA-MSPD with VF-MSPD and VA-MSPD method. The errors bars represent RSD $(n=3)$.

\section{Vibration Frequency of Ball Mill}

Figure 2D showed that the extraction efficiency of target isoflavones was increased as vibration frequency was enhanced from 500 to 800 times per min. However, frequency of 1,000 times per min reduced extraction efficiency of the four isoflavones. Thus, the optimum vibration frequency was set at 800 times/min.

\section{Selection of Methanol With Different Concentration}

Selective and efficient desorption of isoflavones in complex mixtures could be achieved by selecting appropriate solvent polarity. It was found that extraction efficiency of isoflavones increased as methanol/water concentration increased from 50 to $80 \%$ (Figure $2 \mathrm{E}$ ). However, there was a slight decrease in yield with $90 \%$ methanol/water. Thus, methanol-water

TABLE 1 | Linearity, LOD, LOQ, repeatability and recovery.

\begin{tabular}{|c|c|c|c|c|c|c|c|c|}
\hline \multirow[b]{2}{*}{ Component } & \multirow[b]{2}{*}{ Calibration curve } & \multirow[b]{2}{*}{ Test range $(\mu \mathrm{g} / \mathrm{ml})$} & \multirow[b]{2}{*}{$r$} & \multirow[b]{2}{*}{ LOD $(\mu \mathrm{g} / \mathrm{ml})$} & \multirow[b]{2}{*}{$\mathrm{LOQ}(\mu \mathrm{g} / \mathrm{ml})$} & \multirow[b]{2}{*}{ Repeatability (RSD\%) } & \multicolumn{2}{|c|}{ Recovery } \\
\hline & & & & & & & Average (\%) & RSD (\%) \\
\hline Daidzin & $y=8.5072 x-7.7909$ & $0.5-125$ & 0.9994 & 0.13 & 0.5 & 2.75 & 86.1 & 4.74 \\
\hline Genistin & $y=12.248 x-11.247$ & $0.5-125$ & 0.9995 & 0.12 & 0.5 & 2.87 & 90.6 & 4.63 \\
\hline Daidzein & $y=11.532 x-8.4018$ & $0.5-125$ & 0.9997 & 0.25 & 0.8 & 1.83 & 94.8 & 5.00 \\
\hline Genistein & $y=15.683 x-11.247$ & $0.5-125$ & 0.9995 & 0.07 & 0.2 & 1.35 & 93.7 & 4.24 \\
\hline
\end{tabular}


TABLE 2 | Accuracy and precison of intra-day and inter-day and stability for $24 \mathrm{~h}$.

\begin{tabular}{|c|c|c|c|c|c|c|c|}
\hline \multirow{2}{*}{ Component ( $\mu \mathrm{g} / \mathrm{ml})$} & \multirow{2}{*}{ Concentration } & \multicolumn{2}{|c|}{ Intra-day } & \multicolumn{2}{|c|}{ Inter-day } & \multicolumn{2}{|c|}{ Stability for $24 \mathrm{~h}$} \\
\hline & & Accuracy (\%) & RSD (\%) & Accuracy (\%) & RSD (\%) & Remain (\%) & RSD (\%) \\
\hline \multirow[t]{3}{*}{ Daidzin } & 2 & 103 & 2.08 & 105 & 1.19 & 104 & 2.16 \\
\hline & 5 & 104 & 4.25 & 105 & 2.31 & 104 & 3.41 \\
\hline & 50 & 100 & 1.50 & 105 & 3.77 & 103 & 1.46 \\
\hline \multirow[t]{3}{*}{ Genistin } & 2 & 102 & 1.66 & 104 & 1.06 & 103 & 1.73 \\
\hline & 5 & 102 & 3.89 & 105 & 2.22 & 104 & 2.87 \\
\hline & 50 & 97.0 & 1.47 & 100 & 1.39 & 98.7 & 1.36 \\
\hline \multirow[t]{3}{*}{ Daidzein } & 2 & 94.8 & 3.38 & 100 & 1.83 & 97.30 & 1.96 \\
\hline & 5 & 103 & 1.97 & 96.5 & 1.10 & 99.1 & 3.09 \\
\hline & 50 & 96.2 & 0.64 & 96.1 & 1.61 & 96.2 & 1.56 \\
\hline \multirow[t]{3}{*}{ Genistein } & 2 & 102 & 1.35 & 102 & 1.35 & 102 & 1.58 \\
\hline & 5 & 102 & 4.82 & 102 & 4.32 & 102 & 4.85 \\
\hline & 50 & 102 & 3.07 & 105 & 3.77 & 103 & 2.87 \\
\hline
\end{tabular}

$(80 / 20, \mathrm{v} / \mathrm{v})$ was the best eluting solvent for VVA-MSPD procedure.

\section{Volume of Eluting Solvent}

Besides the choice of suitable solvent, moderate volume of eluting solvent during the desorption in VVA-MSPD procedure is also needed. The highest contents of all isoflavones were obtained when an elution volume was $500 \mu \mathrm{L}$ (Figure 2F).

\section{Vortex Time}

Transfer of the target components from solid phase to liquid phase could be accomplished by using optimum vortex. Figure 2G showed that each isoflavone had the highest peak at $3 \mathrm{~min}$ whereas extraction yields declined at $4 \mathrm{~min}$ vortex time. Finally, vortex time was set at $3 \mathrm{~min}$.

\section{Method Validation}

To verify the feasibility of the established VVA-MSPD coupled with HPLC-DAD method for determination of isoflavones in SSP, some parameters including limits of detection (LODs), limits of quantitation (LOQs), linearity, precision, repeatability, stability and recovery were estimated (Tables 1, 2). The established analytical technique was very sensitive with LODs (signal to noise ratio of 3:1) and LOQs (signal to noise ratio of 10:1) ranging from 0.25 to $0.40 \mu \mathrm{g} / \mathrm{ml}$ and $0.80-1.25 \mu \mathrm{g} / \mathrm{ml}$, respectively. Good linearity $(r>0.9994)$ was achieved within the range $0.5-125 \mu \mathrm{g} / \mathrm{ml}$. Intra-day precision at three level concentrations $(2,5,50 \mu \mathrm{g} / \mathrm{ml})$ ranged from 94.8 to $104 \%$ for accuracy and theirs RSDs were below $4.82 \%$ RSDs whereas inter-day precision results were $96.5-105 \%$ for accuracy and RSDs were below $4.32 \%$. The sample stabilities varied from 96.2 to $104 \%$ and theirs RSDs were below $4.85 \%$. The RSDs of repeatability was lower than $2.87 \%$. The recoveries of target components ranged from 86.1 to $94.8 \%$ and theirs RSDs were below $5.00 \%$. These results demonstrated that the proposed method could simultaneously quantify the four isoflavones in the sample of SSP.

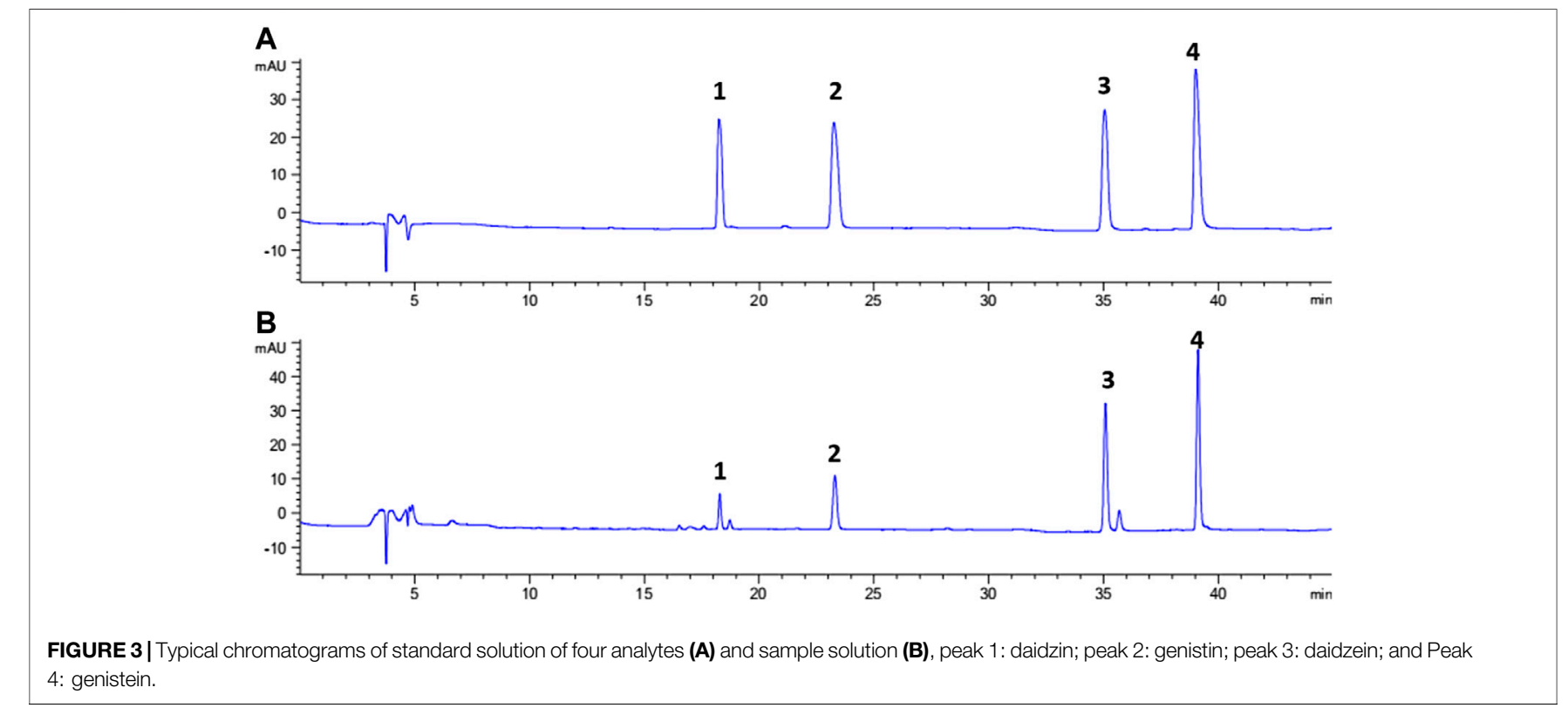


TABLE 3 | Contents of four components in semen sojae praeparatum samples by WA-MSPD, Ultrasound and Heating reflux method $(\mathrm{mg} / \mathrm{g}, n=3)$.

\begin{tabular}{lcccc} 
samples & Daidzin & Genistin & Daidzein & Genistein \\
\hline 1 & $0.21 \pm 0.01$ & $0.31 \pm 0.01$ & $0.72 \pm 0.01$ & $0.76 \pm 0.00$ \\
$1^{\mathrm{a}}$ & $0.14 \pm 0.00$ & $0.22 \pm 0.00$ & $0.59 \pm 0.01$ & $0.54 \pm 0.01$ \\
$1^{\mathrm{b}}$ & $0.11 \pm 0.00$ & $0.17 \pm 0.00$ & $0.49 \pm 0.01$ & $0.44 \pm 0.01$ \\
$2^{2}$ & $0.29 \pm 0.00$ & $0.46 \pm 0.01$ & $0.32 \pm 0.00$ & $0.47 \pm 0.00$ \\
3 & $0.79 \pm 0.01$ & $1.30 \pm 0.03$ & $2.69 \pm 0.05$ & $2.41 \pm 0.02$ \\
4 & $0.88 \pm 0.02$ & $1.17 \pm 0.04$ & $2.07 \pm 0.10$ & $1.08 \pm 0.05$ \\
5 & $1.56 \pm 0.04$ & $1.73 \pm 0.04$ & $1.22 \pm 0.05$ & $0.82 \pm 0.02$
\end{tabular}

aUltrasound extraction.

${ }^{b}$ Heating reflux extraction.

\section{Comparing VVA-MSPD to Other MSPD Methods}

Figure 2H illustrates the extraction efficiencies of the developed VVA-MSPD, VF-MSPD and VA-MSPD methods. Individual peak areas as well as the total contents of all four isoflavones were the highest with our proposed VVA-MSPD method. Comparison with VVA -MSPD and VA-MSPD revealed that homogenization procedure of crushing with ball mill yields better extraction efficiency compared to hand grinding. Moreover, unlike VF-MSPD method, there was no quantitative loss of isoflavones with our developed method due to the absence of a transfer step. Comparatively, extraction efficiency of VVAMSPD was higher than that of VF-MSPD.

\section{Sample Analysis and Comparison of VVA-MSPD With Traditional Extraction Methods}

Representative chromatograms showing the separation of the isoflavones and their contents in each batch of SSP are presented in Figure 3 and Table 3, respectively. Contents of four isoflavones in the five batches of different samples ranged from 0.21 to $1.56 \mathrm{mg} / \mathrm{g}$ for daidzin, $0.31-1.73 \mathrm{mg} / \mathrm{g}$ for genistin, $0.32-2.69 \mathrm{mg} / \mathrm{g}$ for daizein, and $0.48-2.41 \mathrm{mg} / \mathrm{g}$ for genistein, respectively. Furthermore, it was found that VVAMSPD was higher than other conventional extraction methods such as ultrasonic assisted (Niu et al., 2010) and heating reflux (Chai et al., 2014) according to extraction efficiencies for all isoflavones in Samples 1, 1a, and 1b. Comparing to the corresponding yields by ultrasonic assisted extraction, extraction yields of daidzin, genistin daidzein and genistein were increased by $50 \%, 41 \%, 22 \%$ and $41 \%$ usingVVA-MSPD, respectively. The yields of daidzin, genistin daidzein and genistein by VVA MSPD extraction were 1.91, 1.82, 1.47 and 1.73 fold higher than those of daidzin, genistin daidzein and genistein by heating reflux method, respectively. It was concluded that the newly established VVA-MSPD method had outstanding extraction abilities for isoflavones in SSP. Genistein, genistin, daidzin, and daidzein are the main isoflavones of SSP. Among them, genistein is an effective anticancer substance, which could prevent, delay or block the occurrence of cancer through its multi-effect mechanism (Russo et al., 2016). Genistin has a variety of therapeutic effects, such as reducing the risk of osteoporosis and postmenopausal symptoms, anti-inflammatory, anticancer, heart protection, and antioxidant, etc (Liang et al., 2018; Islam et al., 2020). Daidzin could stimulate glucose uptake and has a hypoglycemic effect (Meezan et al., 2005). Daidzein has a protective effect on liver by inhibiting inflammation and oxidative stress and improving lipopolysaccharide induced hepatocyte injury ( $\mathrm{Yu}$ et al., 2020). Therefore, it was of great significance to establish the efficient extraction and quantitative determination method of these bioactive isoflavones. The newly established VVAMSPD method could provide an effective reference for the improvement of the quality standard of SSP.

\section{CONCLUSION}

A rapid, high throughput and effective VVA-MSPD based on SBA-3 method was successfully developed and extracted four isoflavones in SSP. Crushing sample by ball mill was introduced as a homogenization procedure whereas vortex agitation was used as SPE step in MSPD method. Combination of these two procedures ensured that all extraction processes occurred in the same tube. The quantitative loss of isoflavones was eliminated during transfer step as well as extraction efficiency was improved in the extraction procedure. It was demonstrated that VVA-MSPD coupled with high performance liquid chromatography is a rapid, high throughput and efficient method for extracting and determining the isoflavones and is a useful tool for quality evaluation of SSP.

\section{DATA AVAILABILITY STATEMENT}

All datasets presented in this study are included in the article/ supplementary material.

\section{AUTHOR CONTRIBUTIONS}

YC, JL, and XG designed the experiment. YC and XY analyzed the experimental data. AS, XY, EB, JL, and $\mathrm{JH}$ performed the experiment. YC and XY wrote the manuscript.

\section{FUNDING}

This research was supported by the National Key R\&D Program of China (2019YFC1711000), National Natural Science Foundation of China (81973704 and 81374050), Science and Technology Program of Tianjin (No. 19ZYPTJC00060). 


\section{REFERENCES}

Akbari, S., Abdurahman, N. H., and Yunus, R. M. (2019). Optimization of saponins, phenolics, and antioxidants extracted from fenugreek seeds using microwave-assisted extraction and response surface methodology as an optimizing tool. Comptes Rendus Chimie 22(11-12), 714-727. doi:10.1016/j. crci.2019.07.007

Azarkohan, A., Shemirani, F., Alvand, M., and Blin, J. L. (2013). Fast analysis of water samples for trace amount of crystal violet dye based on solid phase extraction using nanoporous SBA-3 prior to determination by fiber opticlinear array detection spectrophotometry. J. Chem. 2013, 1. doi:10.1155/ 2013/530843

Barker, S. A., Long, A. R., and Short, C. R. (1989). Isolation of drug residues from tissues by solid phase dispersion. J. Chromatogr. A 475, 353-361. doi:10.1016/ S0021-9673(01)89689-8

Caldas, S. S., Bolzan, C. M., Menezes, E. J. D., Escarrone, A. L. V., Martins, C. M. G., Bianchini, A., et al. (2013). A vortex-assisted MSPD method for the extraction of pesticide residues from fish liver and crab hepatopancreas with determination by GC-MS. Talanta 112, 63-68. doi:10.1016/j.talanta.2013.03. 054

Cederroth, C. R., and Nef, S. (2009). Soy, phytoestrogens and metabolism: a review. Mol. Cell. Endocrinol. 304 (1-2), 30-42. doi:10.1016/j.mce.2009.02.027

Chai, C., Cui, X. B., Dai, Z. L., Yu, S., Shan, C. X., Qi, H. W., et al. (2014). Content determination of isoflavones in semen sojae praeparatum and the optimization of the fermentation process. Chin. J. Exp. Trad. Med. Formulae 20, 72-76. doi:10.1109/CCM.2003.1194327

Chai, C., Cui, X., Shan, C., Yu, S., and Wen, H. (2017). Contents variation analysis of free amino acids, nucleosides and nucleobases in semen sojae praeparatum fermentation using UFLC-QTRAP MS. Biomed. Chromatogr. 31, e3985. doi:10. 1002/bmc.3985

Chai, C., Cui, X., Shan, C., Yu, S., Wang, X., and Wen, H. 2019). Simultaneous characterization and quantification of varied ingredients from sojae semen praeparatum in Fermentation Using UFLC-TripleTOF MS. Molecules 24(10), 1864. doi:10.3390/molecules24101864

Chen, K. L., Bi, K. S., Han, F., Zhu, H. Y., and Yin, R. (2015). Evaluation of the protective effect of Zhi-Zi-da-Huang decoction on acute liver injury with cholestasis induced by a-naphthylisothiocyanate in rats. J. Ethnopharmacol. 172. doi:10.1016/j.j-ep.2015.06.04310.1016/j.jep.2015.06.043

Chen, Y.-H., Chang, C.-Y., and Ding, W.-H. (2016a). Vortex-homogenized matrix solid-phase dispersion for the extraction of short chain chlorinated paraffins from indoor dust samples. J. Chromatogr. A 1472, 129-133. doi:10.1016/j. chroma.2016.10.048

Chen, J.-M., Yang, C.-C., Chung, W.-H., and Ding, W.-H. (2016b). Vortexhomogenized matrix solid-phase dispersion coupled with gas chromatography-electron-capture negative-ion mass spectrometry to determine halogenated phenolic compounds in seafood. RSC Adv., 6, 96510. doi:10.1039/c6ra20680h

Chinese Pharmacopoeia Commission (2020). Pharmacopoeia of the People's Republic of China, Vol. 1. Beijing, China: China Medical Science and Technology Press.

Chung, W. H., Lin, J. S., and Ding, W. H. (2019). Dual-vortex-assisted matrix solidphase dispersion coupled with isotope-dilution ultrahigh-performance liquid chromatography-high resolution mass spectrometry for the rapid determination of parabens in indoor dust samples. J. Chromatogr. A. 1605, 460367. doi:10.1016/j.chroma.2019.4 60367

Dorota, W., and Marta, G. (2019). New insights into the application of MSPD in various fields of analytical chemistry. Trac. Trends Anal. Chem. 112, 29-51. doi:10.1016/j.trac.2018.12.028

Du, K. Z., Li, J., Bai, Y, An, M. G., Gao, X. M., and Chang, Y. X. (2018). A green ionic liquid-based vortex-forced MSPD method for the simultaneous determination of 5-HMF and iridoid glycosides from Fructus Corni by ultrahigh performance liquid chromatography. Food Chem. 244, 190-196. doi:10.1016/j.foodchem.2017.10.0.057

Escarrone, A. L. V., Caldas, S. S., Soares, B. M., Martins, S. E., Primel, E. G., and Maia Nery, L. E. (2014). A vortex-assisted MSPD method for triclosan extraction from fish tissues with determination by LC-MS/MS. Anal. Methods 6, 8306-8313. doi:10.1039/c4ay01518e
Guo, H., Zhang, Z., Yao, Y., Liu, J., Chang, R., Liu, Z., et al. (2018). A new strategy for statistical analysis-based fingerprint establishment: application to quality assessment of Semen sojae praeparatum. Food Chem. 258, 189-198. doi:10. 1016/.foo-dchem.2018.03.06710.1016/j.foodchem.2018.03.067

Hertzog, G. I., Soares, K. L., Caldas, K. L., and Primel, E. G. (2015). Study of vortexassisted MSPD and LC-MS/MS using alternative solid supports for pharmaceutical extraction from marketed fish. Anal. Bioanal. Chem. 407(16), 4793-4803. doi:10.1007/s00 216-015-8685-3

Islam, A., Islam, M., Hasan, M., and Akanda, M. (2020). The potential health benefits of the isoflavone glycoside genistin. Arch Pharm. Res. 434, 14-63. doi:10.1007/s12 272-020-01233-2

Kemmerich, M., Demarco, M., Bernardi, G., Prestes, O. D., Adaime, M. B., and Zanella, R. (2019). Balls-in-tube matrix solid phase dispersion (BiT-MSPD): an innovative and simplified technique for multiresidue determination of pesticides in fruit samples. J. Chromatogr. A. 110, 395-401. doi:10.1016/j. chroma.2019.460640

Liang, Y., Zhao, W., Wang, C., Wang, Z., Wang, Z., and Zhang, J. (2018). A comprehensive screening and identification of genistin metabolites in rats based on multiple metabolite templates combined with UHPLC-HRMS analysis. Molecules 23, 1862. doi:10.3390/molecules23081862

Meezan, E., Meezan, E. M., Jones, K., Moore, R., Barnes, S., and Prasain, J. K. (2005). Contrasting effects of puerarin and daidzin on glucose homeostasis in mice. J. Agric. Food Chem. 53, 8760-8767. doi:10.1021/jf058105e

Míguez-Framil, M., Cabarcos, P., Tabernero, M. J., Bermejo, A. M., BermejoBarrera, P., and Moreda-Piñeiro, A. (2013). Matrix solid phase dispersion assisted enzymatic hydrolysis as a novel approach for cocaine and opiates isolation from human hair. J. Chromatogr. A 1316, 15-22. doi:10.1016/j. chroma.2013.09.063

Mocan, A., Carradori, S., Locatelli, M., Secci, D., Cesa, S., Mollica, A., et al. (2018) Bioactive isoflavones from Pueraria lobata root and starch: different extraction techniques and carbonic anhydrase inhibition. Food Chem. Toxicol. 112, 441-447. doi:10.1016/j.fct.2017.08.009

Moreda-Pineiro, J., Alonso-Rodriguez, E., Romaris-Hortas, V., Moreda-Pineiro, A., Lopez-Mahia, P., Muniategui-Lorenzo, S., et al. (2012). Assessment of the bioavailability of toxic and non-toxic arsenic species in seafood samples. Food Chem. 130, 552-560. doi:10.1016/j.foodchem.2011.07.071

Niu, L. Y., Shi, S. Q., Liu, M. Y., Wang, F., and Wang, X. G. (2010). Processing of semen sojae praeparatum. Chin. Trad. Patent Med. 32, 1372-1376. doi:10. 19540/j.cnki.cjcmm.20190321.301

Qu, K. K., Zhao, L. S., Luo, X. Y., Zhang, C. N., Hou, Y. P., Bi, K. S., et al. (2014). An LC-MS method for simultaneous determination of five iridoids from Zhi-zi-chi Decoction in rat brain microdialysates and tissue homogenates: towards an in depth study for its antidepressive activity. J. Chromatogr. B 965, 206-215 doi:10. 1016/j.jchromb. 2014.03.032

Qu, L. P., Fan, G. R., Peng, J. Y., and Mi, H. M. (2007). Isolation of six isoflavones from Semen sojae praeparatum by preparative HPLC. Fitoterapia 78, 200-204. doi:10.1016/j.fitote. 2006.11.002

Russo, M., Russo, G. L., Daglia, M., Kasi, P. D., Ravi, S., Nabavi, S. F., et al. (2016). Understanding genistein in cancer: the "good" and the "bad" effects: a review. Food Chem. 196, 589-600. doi:10.1016/j.foodchem.2015.09.085

Sun, S., Yao, K., Zhao, S., Zheng, P., Wang, S., Zeng, Y., et al. (2018). Determination of aflatoxin and zearalenone analogs in edible and medicinal herbs using a group-specific immunoaffinity column coupled to ultra-high-performance liquid chromatography with tandem mass spectrometry. J. Chromatogr. B, 1092, 228-236. doi:10.1016/j.jchromb.2018.06.012

Vitale, D. C., Piazza, C., Melilli, B., Drago, F., and Salomone, S. (2013). Isoflavones: estrogenic activity, biological effect and bioavailability. Eur. J. Drug Metab. Pharmacokinet. 38 (1), 15-25. doi:10.1007/s13318-012-0112-y

Wang, H. Q., Zhu, Y. X., Liu, Y. M., Wang, R. L., and Wang, S. F. (2019). Rapid discovery and identification of the anti-inflammatory constituents in Zhi-Shi-Zhi -Zi-Chi-Tang. Nat. Med. China 17, 308-320. doi:10.1016/s1875-5364(19)30035-4

Wang, J., Shi, Q., Wu, C., and Feng, F. (2016). Dynamic metabolic profile of Zhi-ZiDa-Huang decoction in rat urine based on hybrid liquid chromatography-mass spectrometry coupled with solid phase extraction. J. Chromatogr. B 1036-1037, 100-113. doi:10.1016/j.jchromb.2016.10.003

Wu, X., Zhu, B. H., Lu, L., Huang, W., and Pang, D. (2012). Optimization of a solid phase extraction and hydrophilic interaction liquid chromatography-tandem mass spectrometry method for the determination of metformin in dietary 
supplements and herbal medicines. Food Chem. 133, 482-488. doi:10.1016/j. foodchem10.1016/j.foodchem.2012.01.005

Xiong, A.-Z., Yang, L., Zhang, F., Yang, X.-J., Wang, C.-H., and Wang, Z.-T. (2009). Determination of total retronecine esters-type hepatotoxic pyrrolizidine alkaloids in plant materials by pre-column derivatization high-performance liquid chromatography. Biomed. Chromatogr. 23, 665-671. doi:10.1002/bmc.1172

Xiong, H., Gong, X., and Qu, H. (2012). Monitoring batch-to-batch reproducibility of liquid-liquid extraction process using in-line near-infrared spectroscopy combined with multivariate analysis. J. Pharm. Biomed. Anal. 70, 178-187. doi:10.1016/j.jpba.2012.06.028

Yang, X., Yang, L., Xiong, A., Li, D., and Wang, Z. (2011). Authentication of Senecio scandens and $S$. vulgaris based on the comprehensive secondary metabolic patterns gained by UPLC-DAD/ESI-MS. J. Pharm. Biomed. Anal. 56, 165-172. doi:10.1016/j.jpba.2011.05.004

Yao, Y., Ma, X., Li, T., Guo, H., Chang, R., Liu, J., et al. (2018). Quantification of isoflavone glycosides and aglycones in rat plasma by LC-MS/MS: troubleshooting of interference from food and its application to pharmacokinetic study of Semen Sojae Praeparatum extract. J. Pharm. Biomed. Anal. 161, 444. doi:10.1016/j.jpba.2018.09.011
Yu, Z., Yang, L., Deng, S., and Liang, M. (2020). Daidzein ameliorates LPS-induced hepatocyte injury by inhibiting inflammation and oxidative stress. Eur. J. Pharmacol. 885, 173399. doi:10.1016/j.ejphar.2020.173399

Yue, Y., Qiu, Z.-D., Qu, X.-Y., Deng, A.-P., Yuan, Y., Huang, L.-Q., and Lai, C.-J. -S. (2018). Discoursing on Soxhlet extraction of ginseng using association analysis and scanning electron microscopy. Journal of Pharmaceutical Analysis 8, 312-317. doi:10.1016/j.jpha.2018.08.003

Conflict of Interest: The authors declare that the research was conducted in the absence of any commercial or financial relationships that could be construed as a potential conflict of interest.

Copyright (C) 2020 Yang, Sun, Boadi, Li, He, Gao and chang. This is an open-access article distributed under the terms of the Creative Commons Attribution License (CC $B Y)$. The use, distribution or reproduction in other forums is permitted, provided the original author(s) and the copyright owner(s) are credited and that the original publication in this journal is cited, in accordance with accepted academic practice. No use, distribution or reproduction is permitted which does not comply with these terms. 